\title{
Perceived confidence in the FAST exam before and after an educational intervention in a developing country
}

\author{
Andre K. Crouch • Matthew Dawson • Deanne Long • \\ Derrick Allred • Troy Madsen
}

Received: 30 July 2009 /Accepted: 23 November 2009/Published online: 27 February 2010

(C) Springer-Verlag London Ltd 2010

\begin{abstract}
Background Trauma care in developing countries suffers from many limitations related to equipment shortages, disrepair, quality assurance, and lack of training. Health care providers in the three principal hospitals in Cusco, Peru have ultrasound machines, but they do not utilize this for the focused assessment of sonography in trauma (FAST) scan (only one of the three hospitals has a computed tomography scanner).

Aims The goal of this study was to assess the confidence of physicians in a developing country to conduct a FAST exam after an educational intervention.

Methods Participants were Peruvian health care workers who attended a 2-day conference on trauma. Participants completed a questionnaire based on a 5-point Likert scale ( $1=$ no confidence, $5=$ high confidence $)$ to assess comfort with the FAST scan before and after a FAST teaching workshop, which included hands-on ultrasound training. Thirteen individuals, eight of whom were physicians, completed the training and survey. Results were analyzed using paired $t$ test statistics and are reported as pre- and post-training mean scores ( \pm standard error), with $p<0.05$ considered statistically significant.

Results Participants rated their confidence in using the FAST exam on a trauma patient with an average score of $3.3( \pm 0.3)$ pre-training and $4.5( \pm 0.2)$ post-training $(p=0.007)$. When asked about their comfort level in making clinical decisions
\end{abstract}

A. K. Crouch $\cdot$ M. Dawson $\cdot$ D. Long $(\bowtie) \cdot$ D. Allred $\cdot$ T. Madsen Division of Emergency Medicine, University of Utah,

30 N. 1900 E. 1C26,

Salt Lake City, UT 84132, USA

e-mail: deanne.long@hsc.utah.edu based on the FAST scan, pre-training average score was 3.5 $( \pm 0.4)$ and post-training was $4.5( \pm 0.2), p=0.016$. Participants also answered questions about their comfort with the technical aspects of using the ultrasound machine: ability to choose the correct probe (pre: 3.9 , post: $4.6, p=$ 0.011 ), choosing the correct probe orientation (pre: 3.9, post: 4.6, $p=0.008$ ), and adjusting the depth and gain (pre: 3.1, post: $4.4, p=0.001$ ). Finally, participants rated their comfort with the specific views of the FAST scan: ability to find the correct subcostal view (pre: 3.3 , post: $4.9, p<0.001$ ), right upper quadrant view (pre: 3.2, post: 4.6, $p<0.001$ ), left upper quadrant view (pre: 3.2 , post: $4.4, p=0.001$ ), and the pelvic view (pre: 3.2 , post: $4.5, p<0.001$ ).

Conclusion After a training session in the use of ultrasound in trauma, health care workers in Cusco, Peru reported increased confidence in their FAST scan ability and in their comfort in using this exam for clinical decision-making. Future research should include objective testing of participants' skill as well as longitudinal follow-up to determine the extent to which the FAST scan has been incorporated into participants' evaluations of trauma patients.

Keywords Ultrasound - International medicine - Education

\section{Introduction}

Trauma is a leading cause of death and disability worldwide, but trauma care in developing countries suffers from many limitations. Most of these challenges relate to equipment shortages, disrepair, quality assurance, and training. The focused assessment of sonography in trauma (FAST) is an area that has been identified as lacking in many developing countries. In multiple settings it has been demonstrated that an ultrasound machine is available, but due to lack of training it is 
not utilized [1]. Indeed, the Guidelines for Essential Trauma Care published by the World Health Organization (WHO) consider ultrasound availability desirable as long as there is availability of skilled staff [2]. Meeting these criteria is a challenge as previously shown in Brazil, a relatively advanced developing country [3].

In Cusco, Peru trauma care providers have received little specific training. An ultrasound machine is present but not used because of the lack of provider training. Most hospitals in the city also lack a computed tomography (CT) scanner, which increases the importance of the potential use of ultrasound to evaluate for hemoperitoneum. The FAST exam in multiple studies has been shown to have similar accuracy to diagnostic peritoneal lavage (DPL) $[4,5]$, but it is noninvasive, requires less time, and has no contraindications. In addition, training for 2-8 h has been shown to be adequate to teach trauma care providers the FAST exam [6-8].

Our goal was to teach Peruvian health care providers the FAST exam during a 2-day conference on trauma. In order to measure progress we used a survey to measure their confidence with the FAST before and after training. A confidence survey was used because previous studies have demonstrated that operator confidence correlates with increased accuracy in abdominal ultrasound [9]. Furthermore, more accurate abdominal ultrasounds presumably have the potential to aid diagnosis and treatment decisions in this community where a CT scanner is not available in most hospitals, but in which providers have access to an ultrasound.

\section{Methods}

In April 2008, physicians from the University of Utah Medical Center held a 2-day conference on trauma in Cusco, Peru, with the goal of standardizing and improving trauma care in the region. Part of the conference was aimed at teaching the FAST exam and measuring the participants' confidence level with the exam. The FAST exam was taught during a 2.5 -h workshop with hands-on teaching. An emergency medicine resident who is proficient in the FAST exam (AC) presented a 1-h lecture with Spanish translation on the basic principles of ultrasound. The lecture included a description of the four standard views obtained during a FAST scan with examples of positive scans. After the didactic, participants spent $1.5 \mathrm{~h}$ practicing the ultrasound exam on volunteers using a GE Logiq e and an ultrasound machine from the emergency department of the hosting hospital. The participants consisted of approximately 25 health care providers, comprising local physicians and nurses from various hospitals and clinics in Cusco, Peru and the surrounding communities.
Participants voluntarily completed a questionnaire based on a 5-point Likert scale $(1=$ no confidence, $5=$ high confidence) to assess comfort with the FAST scan before and after the workshop. Thirteen individuals, eight of whom were physicians, completed the training and survey. The survey was completed in Spanish, but has been included in this paper in English (Table 1).

Results were analyzed using paired $t$ test statistics and are reported as pre- and post-training mean scores $( \pm$ standard error), with $p<0.05$ considered statistically significant.

\section{Results}

FAST workshop participants reported significant increases in their confidence in each aspect surveyed. Participants rated their confidence in using the FAST exam on a trauma patient with an average score of 3.3 pre-training and 4.5 post-training on the 5-point Likert scale $(p=0.007)$. When asked about their comfort level in making clinical decisions based on the FAST scan, pre-training average score was 3.5 and post-training was $4.5(p=0.016)$.

Participants also answered questions about their comfort with the technical aspects of using the ultrasound machine: ability to choose the correct probe (pre: 3.9 , post: $4.6, p=$ 0.011 ), choosing the correct probe orientation (pre: 3.9, post: 4.6, $p=0.008$ ), and adjusting the depth and gain (pre: 3.1, post: $4.4, p=0.001$ ).

Finally, participants rated their comfort with the specific views of the FAST scan: ability to find the correct subcostal view (pre: 3.3 , post: $4.9, p<0.001$ ), right upper quadrant view (pre: 3.2, post: 4.6, $p<0.001$ ), left upper quadrant view (pre: 3.2 , post: $4.4, p=0.001$ ), and the pelvic view (pre: 3.2 , post: $4.5, p<0.001$ ) (Table 2).

\section{Limitations}

This study has several limitations. Only 13 of the participants completed the survey. Those who felt more confident in their skill may have been more willing to complete the survey. As such, however, the survey does provide a sense of the response among the majority of the workshop participants.

Another limitation of the study was the lack of objective measure of participants' ability to perform the FAST scan. It is not clear what technical ability or accuracy corresponds with a confidence level of 3 or how this differs from a confidence level of 4. Furthermore, it is unclear if this represents a clinically significant improvement. Objective technical skill data were difficult to obtain because of time constraints with the vast amount of information being taught during the short 2-day course. However, it has been 
Table 1 Survey used to evaluate participant subjective comfort and skill

Level of training and specialty (Ex: surgeon, nurse, health care worker):

FAST Questionnaire

Please complete the following questionnaire on a scale of 1-5. 1 being not at all confident, and 5 being extremely confident.

1. Do you feel comfortable choosing the correct probe for a FAST exam?

2. Do you feel you can choose the correct orientation of the probe?

$$
\begin{array}{lllll}
1 & 2 & 3 & 4 & 5
\end{array}
$$

3. Do you feel comfortable adjusting the gain and depth to maximize picture quality?

$\begin{array}{lllll}1 & 2 & 3 & 4 & 5\end{array}$

4. How do you feel visualizing the subcostal view?

$$
\begin{array}{lllll}
1 & 2 & 3 & 4 & 5
\end{array}
$$

5. How do you feel visualizing the right upper quadrant view?

$$
\begin{array}{lllll}
1 & 2 & 3 & 4 & 5
\end{array}
$$

6. How do you feel visualizing the left upper quadrant view?

$$
\begin{array}{lllll}
1 & 2 & 3 & 4 & 5
\end{array}
$$

7. How do you feel visualizing the pelvic view?

$$
\begin{array}{lllll}
1 & 2 & 3 & 4 & 5
\end{array}
$$

8. How confident would you feel doing a FAST exam on a trauma patient?

$$
\begin{array}{lllll}
1 & 2 & 3 & 4 & 5
\end{array}
$$

9. How comfortable would you feel making clinical decisions based on the results of your FAST exam?

$$
\begin{array}{lllll}
1 & 2 & 3 & 4 & 5
\end{array}
$$

shown in a previous study that operator confidence does in fact correlate with more accurate ultrasounds [4].

The study also was performed at one site over a short 2-day course with no long-term data on provider skill and confidence available at this time. However, it has been objectively shown that novice ultrasonographers can learn the FAST scan during a short 2-day trauma course [8]. Also, it has been demonstrated that in a developing country (Rwanda in 2008) after ultrasound instructors leave, providers who were taught the new modality continue to use the new skill with great frequency and accuracy [10].

\section{Conclusion}

The FAST scan is an important part of a trauma evaluation with the ability to affect patient care and decision-making. This importance and impact is greatly amplified in a setting where resources are limited and no CT scanner is available.

An important precursor to using the FAST scan correctly is having hands-on training and developing the confidence to use it and make decisions in a clinical setting. We have demonstrated with our FAST workshop and confidence surveys that trauma providers in a developing country

Table 2 Participant pre- and post-training evaluation of FAST confidence and skill

\begin{tabular}{llll}
\hline Survey question & Pre-workshop confidence & Post-workshop confidence & $p$ value \\
\hline Overall confidence using FAST scan on a trauma patient & 3.3 & 4.5 & 4.6 \\
Ability to choose correct probe & 3.9 & 4.6 & 0.007 \\
Ability to choose correct probe orientation & 3.9 & 4.4 & 0.011 \\
Ability to adjust depth and gain & 3.1 & 4.9 & 4.6 \\
Ability to find subcostal view & 3.3 & 4.4 & $<.008$ \\
Ability to find RUQ view & 3.2 & 4.5 & $<0.001$ \\
Ability to find LUQ view & 3.2 & 4.5 & 0.001 \\
Ability to find pelvic view & 3.2 & 3.5 & $<.001$ \\
Confidence in making clinical decisions based on FAST scan & 3.5 & 0.016 \\
\hline
\end{tabular}

$R U Q$ right upper quadrant, $L U Q$ left upper quadrant 
reported increased confidence in FAST scanning with a brief educational intervention.

More research including skill retention, more participants, translation into practice, and objective measures of skill is needed. However, this study may provide guidance to practitioners in performing educational interventions to improve trauma ultrasound in developing countries with limited resources.

\section{References}

1. Mock C, Nguyen S, Quansah R et al (2006) Evaluation of trauma care capabilities in four countries using the WHO-IATSIC Guidelines for Essential Trauma Care. World J Surg 30:946-956

2. Mock C, Lormand JD, Goosen J et al (2004) Guidelines for essential trauma care. World Health Organization, Geneva

3. Banta HD (1986) Medical technology and developing countries: the case of Brazil. Int J Health Serv 16:363-373

4. Brooks A, Davies B, Smethhurst M, Connolly J (2004) Prospective evaluation of non-radiologist performed emer gency abdominal ultrasound for haemoperitoneum. Emerg Med J 21:e5

5. Hsu JM, Joseph AP, Tarlinton LJ et al (2007) The accuracy of focused assessment with sonography in trauma (FAST) in blunt trauma patients: experience of an Australian major trauma service. Injury 38:71-75

6. Abu-Zidan FM, Dittrich K, Czechowski JJ, Kazzam EE (2005) Establishment of a course for focused assessment sonography for trauma. Saudi Med J 26:806-811

7. Melanson SW, Heller MB (2004) Principles of emergency department sonography. In: Tintintinalli JE, Kelen GD, Stapczynski JS (eds) Emergency medicine: a comprehensive study guide, 6th edn. McGraw-Hill, New York, pp 1870-1878

8. Han DC, Rozycki GS, Schmidt JA, Feliciano DV (1996) Ultrasound training during ATLS: an early start for surgical interns. J Trauma 41:208-213

9. Jang T, Naunheim R, Sineff S, Aubin C (2007) Operator confidence correlates with more accurate abdominal ultrasounds by emergency medicine residents. J Emerg Med 33 (2): $175-179$

10. Shah SP, Epino H, Bukhman G, Umulisa I, Dushimiyimana J, Reichman A, Noble VE (2009) Impact of the introduction of ultrasound services in a limited resource setting: rural Rwanda 2008. BMC Int Health Hum Rights 9:4 